\title{
Energy-Efficient Incentives Resource Allocation Scheme in Cooperative Communication System
}

\author{
Zi Yan Liu $\mathbb{D}^{1},{ }^{1}$ Pan Mao, ${ }^{1,2}$ Li Feng, ${ }^{3}$ and Shi Mei Liu ${ }^{1}$ \\ ${ }^{1}$ College of Big Data and Information Engineering, Guizhou University, Guiyang 550025, China \\ ${ }^{2}$ Huaxin Consulting Co., Ltd., Hangzhou 310014, China \\ ${ }^{3}$ State Grid Chongqing Electric Power Company, Chongqing 400014, China
}

Correspondence should be addressed to Zi Yan Liu; gzucomm@gmail.com

Received 21 January 2018; Revised 25 March 2018; Accepted 24 April 2018; Published 5 June 2018

Academic Editor: Zheng Chu

Copyright (C) 2018 Zi Yan Liu et al. This is an open access article distributed under the Creative Commons Attribution License, which permits unrestricted use, distribution, and reproduction in any medium, provided the original work is properly cited.

\begin{abstract}
Appropriate resource allocation has great significance to enhance the energy efficiency (EE) for cooperative communication system. The objective is to allocate the resource to maximize the energy efficiency in single-cell multiuser cooperative communication system. We formulate this problem as subcarrier-based resource allocation and solve it with path planning in graph theory. A twolevel neural network model is designed, in which the users and subcarrier are defined as network nodes. And then we propose an improved intelligent water drops algorithm combined with Genetic Algorithm; boundary condition and initialization rules of path soil quantity are put forward. The simulation results demonstrate that the proposed resource allocation scheme can effectively improve the energy efficiency and enhance QoS performance.
\end{abstract}

\section{Introduction}

The rapid energy consumption due to the demands of mobile communication services has become a subject of global interests from environment perspective. On one hand, because of its slow development and limited capacity, battery technology becomes the bottleneck of limiting the development of the portable terminals [1]; on the other hand, enormous energy consumption of the communication industry indirectly leads to the greenhouse gas emission and increases the operators' operating costs. Statistics show that, in 2009, the power consumption of three service providers in China was 28.9 billion degrees, which equals 4.41 million tons of coal burning. By 2014, the energy consumption had been up to 6.71 million tons, with a $52 \%$ increase in 5 years $[2,3]$. Compared to other industries, it is essential for the communication industry to reduce energy consumption. Meanwhile, as important support for social informatization, there is a very broad prospect for the communication industry to promote energy conservation in the society information industry. Therefore, designing a high energy-efficient communication system has become a consensus of the communication industry.
Cooperative communication $[4,5]$ is defined as follows: in a cell, the adjacent devices with single antenna create a virtual MIMO system by sharing their antennas with each other $[6,7]$ to achieve the goal of overcoming the multipath fading and gaining the benefit of multiantenna space diversity. In cooperative communication system, a reasonable resource allocation scheme has great significance in improving the spectral efficiency and reducing the energy consumption. The traditional design of cooperative communication system mainly focuses on the improvement of system capacity, outage probability, spectral efficiency, and other performances. With the scholars' attention to the energy consumption of communication industry and the concept of green communication, the energy efficiency of cooperative communication system is gaining widespread concern. Wong et al. [8] studied the network-level resource scheduling scheme in cooperative communication system and proposed a cooperative concept to obtain the higher energy efficiency; at the same time they also designed high energy-efficient network architecture. A resource allocation scheme on game theory is established; it is proven that the cooperation between users can effectively improve the energy efficiency of the system [9]. In [10], 


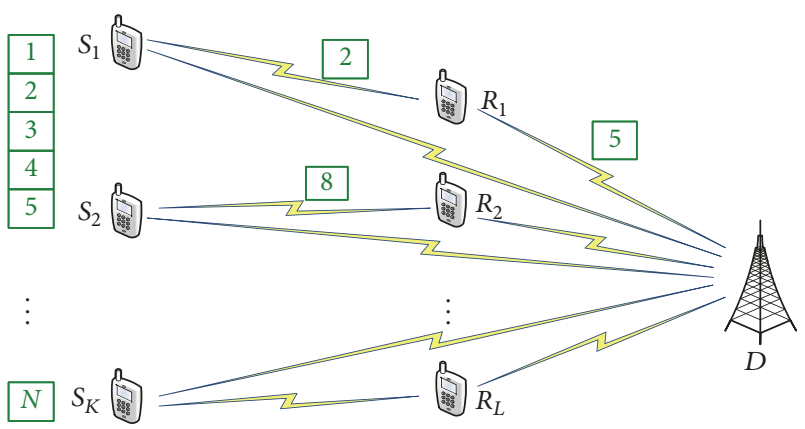

FIGURE 1: Cooperative communication model.

an algorithm is proposed to select the optimal relay in a single-relay cooperation system. Compared with the fixed cooperation method, the energy consumption is significantly reduced. The wireless sensor networks (WSNs) with energy harvesting and cooperative communication were studied in [11], and an energy-efficient scheduling strategy is proposed; the optimal scheduling problem is solved by using a Markov Decision Process (MDP). In [12], the process of collaboration was divided into two time slots. An algorithm of relay selection and power allocation is proposed for the minimum BER and maximum system capacity, respectively. However, these studies have achieved the purpose of improving the energy efficiency from the perspective of energy consumption and have not analyzed the energy efficiency in the form of quantitative indicators. QoS in the actual scenario is not considered, especially the demands of high transmission rate.

In this paper, we consider a single-cell multiuser cooperative communication system. To meet the demands of QoS performance, a two-level neural network model based on intelligent water drops (IWDs) algorithm is proposed to solve the problem of resource allocation with optimal energy efficiency. And then, the IWDs algorithm combined with Genetic Algorithm (GA) is improved to allocate resources flexibly and enhance performance.

This paper is organized as follows. Section 2 introduces the system model as well as the function of the proposed optimal problem. In Section 3, a novel algorithm is proposed. After that, the optimal energy-efficient resource allocation scheme is presented in Section 4. In Section 5, numerical results are depicted. Finally, Section 6 concludes the paper.

\section{System Model and Problem Formulation}

In this section, the proposed system model is presented, followed by the optimization problem.

2.1. System Model. We consider an uplink single-cell multiuser cooperative communication scenario as shown in Figure 1, and the radius is 600 meters. It consists of source node $S$, relay node $R$, and destination node $D$. In particular, the source node is $K$ users with call requests, and $L$ is the relay node that participates in the cooperative communication, and the destination node is the base station (BS). Orthogonal Frequency Division Multiple Access (OFDMA) is the multiple access scheme, and the available bandwidth $B$ is divided into $N$, in which the subcarriers are orthogonal to each other. It is assumed that the relay node has perfect channel state information (CSI). Further, the channels are considered as large-scale and small-scale fading. That is, large-scale fading is defined as path loss and small-scale fading is Rayleigh fading, respectively. Moreover, the forwarding mode is Decodeand-Forward (DF), and the relay network is operated in halfduplex mode.

2.2. Problem Formulation. Assume that the channel states between any two terminals $\left(S_{k}, R_{l}, D\right)$ are independent of each other. $h_{i j}^{n}\left(i, j \in\left\{S_{k}, R_{l}, D\right\}\right)$ indicates the channel fading between the device $i$ and $j$. The channel fading between the nodes can be given by [13]

$$
\begin{aligned}
& E\left(\left|h_{S_{k} D}^{n}\right|^{2}\right)=d_{S_{k} D}^{-\alpha}, \\
& E\left(\left|h_{S_{k} R_{l}}^{n}\right|^{2}\right)=d_{S_{k} R_{l}}^{-\alpha}, \\
& E\left(\left|h_{R_{l} D}^{n}\right|^{2}\right)=d_{R_{l} D}^{-\alpha},
\end{aligned}
$$

where $\left|h_{S_{k} D}^{n}\right|^{2},\left|h_{S_{k} R_{l}}^{n}\right|^{2},\left|h_{R_{l} D}^{n}\right|^{2}$ denote the CSI on the subcarrier $n$, from the user $S_{k}$ to the destination node $D$, from the user $S_{k}$ to the relay $R_{l}$, and from the relay $R_{l}$ to the destination node $D$, respectively. $d_{i j}\left(i, j \in\left\{S_{k}, R_{l}, D\right\}\right)$ represents the distance between the device $i$ and $j . E(\cdot)$ is the average operator, and $\alpha \in[3,5]$ is the channel fading factor.

The energy efficiency is defined as follows [14]:

$$
\eta_{\mathrm{EE}}=\frac{R}{P_{\mathrm{tot}}},
$$

where $R$ represents the total transfer rate and $P_{\text {tot }}$ denotes the total energy consumption.

The transmission procedures of cooperative communication system are divided into two time slots [15]: the first is the broadcast slots, during which the source node $S$ broadcasts the information to the relay node $R$ and the destination node $D$; the second is the forwarding slot, during which the relay node $R$ processes the received signal and forwards it to the destination node $D$. In the case of dynamic allocation of transmission time slot, it is normalized. Particularly, $t$ represents the transmission time in the broadcast slot, and $(1-t)$ denotes the time of the relay forwarding slot. Due to the different environments in which three terminals are located, the channel fadings are independent of each other in two different time slots. Two hops of information (broadcasting and forwarding) occupy different time slots, respectively. Therefore, in two hops, we can use the same subcarriers without considering the interference between them, but the system performance may be limited. So, the subcarriers should be allocated independently in two time slots, which involves the subcarrier pair matching and allocation issue.

The distribution coefficient of subcarrier pairs $C_{m, n}^{k} \epsilon$ $\{0,1\}$ is introduced firstly. $C_{m, n}^{k}=1$ represents the case where subcarrier $m(1 \leq m \leq N)$ is paired with $n(1 \leq n \leq N)$, which is noted as the subcarrier pair $(m, n)$. That is, user $k$ 
transfers the information on the subcarrier $m$ at the first slot and on the subcarrier $n$ at the second slot. On the contrary, $C_{m, n}^{k}=0$ means that the subcarrier $m$ is not paired with $n$.
According to the Shannon's equation, after the user $S_{k}$ cooperates with the relay node $R_{l}$, the transfer rate on the subcarrier pair $(m, n)$ can be written as [16]

$$
\begin{aligned}
& R_{m, n}^{k} \\
& \quad=\min \left\{t B \log _{2}\left(1+\frac{C_{m, n}^{k} \beta_{k, l} P_{S}}{N_{R_{l}}}\left|h_{S_{k} R_{l}}^{m}\right|^{2}\right), t B \log _{2}\left(1+\frac{C_{m, n}^{k} P_{S}}{N_{D}}\left|h_{S_{k} D}^{m}\right|^{2}\right)+(1-t) B \log _{2}\left(1+\frac{C_{m, n}^{k} \beta_{k, l} P_{l}}{N_{R_{l}}}\left|h_{R_{l} D}^{n}\right|^{2}\right)\right\},
\end{aligned}
$$

where $\left|h_{S_{k} R_{l}}^{m}\right|^{2},\left|h_{S_{k} D}^{m}\right|^{2}$, and $\left|h_{R_{l} D}^{n}\right|^{2}$, respectively, represent the channel coefficients on the subcarrier pair $(m, n)$ between the devices $i, j\left(i, j \in\left\{S_{k}, R_{l}, D\right\}\right)$. $\beta_{k, l}$ is the relay selection factor, and $\beta_{k, l}=1$ indicates that the $l$ th relay node participates in the $k$ th user cooperative communication process and vice versa with no participation. The solution of $\beta_{k, l}$ is the problem of relay selection in cooperative communication system; we use the method "dichotomous map" proposed in [17]. $P_{S}$ is the transmitting power of the user node, and $P_{l}$ is the transmitting power of the relay node. Respectively, $N_{R_{l}}, N_{D}$ are the noises at the relay node $R$ and the destination node D.
The transmission consumption of user $k$ can be written as

$$
\begin{aligned}
P^{k}=\left(\zeta\left(t P_{S}+(1-t) \beta_{k, l} P_{l}\right)+P_{\mathrm{CT}}+(\right. & \left.+1) P_{\mathrm{CR}}\right), \\
& k \in\{1,2, \ldots, K\},
\end{aligned}
$$

where $\zeta$ is the reciprocal of the drain efficiency of the power amplifier at the transmitter, $P_{\mathrm{CT}}$ is the fixed circuit power at the transmitter, and $P_{\mathrm{CR}}$ is the fixed circuit power at the receiver. follows:

From (2), the energy efficiency of user $k$ can be defined as

$$
\eta_{\mathrm{EE}}^{k}=\frac{R_{m, n}^{k}}{P^{k}}
$$

and the optimal energy-efficient resource allocation is given by

$$
\begin{aligned}
\eta_{\mathrm{EE}}=\max _{C_{m, n}^{k}} \sum_{k=1}^{K} \sum_{m=1}^{N} \sum_{n=1}^{N}\left(\operatorname { m i n } \left\{t B \log _{2}\left(1+\left(C_{m, n}^{k} \beta_{k, l} P_{S} / N_{R_{l}}\right)\left|h_{S_{k} R_{l}}^{m}\right|^{2}\right), t B \log _{2}\left(1+\left(C_{m, n}^{k} P_{S} / N_{D}\right)\left|h_{S_{k} D}^{m}\right|^{2}\right)\right.\right. \\
\left.+(1-t) B \log _{2}\left(1+\left(C_{m, n}^{k} \beta_{k, l} P_{l} / N_{R_{l}}\right)\left|h_{R_{l} D}^{n}\right|^{2}\right)\right\} \\
\\
\left.\cdot\left(\zeta\left(t P_{S}+(1-t) \beta_{k, l} P_{l}\right)+P_{\mathrm{CT}}+(t+1) P_{\mathrm{CR}}\right)^{-1}\right)
\end{aligned}
$$

s.t. $\quad C 1: C_{m, n}^{k} \in\{0,1\}$;

$$
\begin{aligned}
& \text { C2: } \sum_{m}^{N} \sum_{n}^{N} C_{m, n}^{k}=1 \quad \forall k ; \\
& \text { C3: } \sum_{k}^{K} C_{m, n}^{k} \leq 1 \quad \forall m, n ;
\end{aligned}
$$

$$
\begin{aligned}
& \mathrm{C} 4: 0 \leq P_{S}, P_{l} \leq P_{\max } ; \\
& \mathrm{C} 5: R t_{k, l} \geq R t_{\min },
\end{aligned}
$$

where $\mathrm{C} 1$ is the subcarrier matching and the allocation coefficient, indicating that the subcarriers have two states of cooperative communication and noncooperative communication; C2 means that a subcarrier is assigned to one user only; C3 nidicates that a user selects only one subcarrier to cooperate; C4 is defined as the power limitation between users and relay nodes; and C5 is the transmission rate, and $R t_{\text {min }}$ is the minimum transmission rate, which is QoS. 


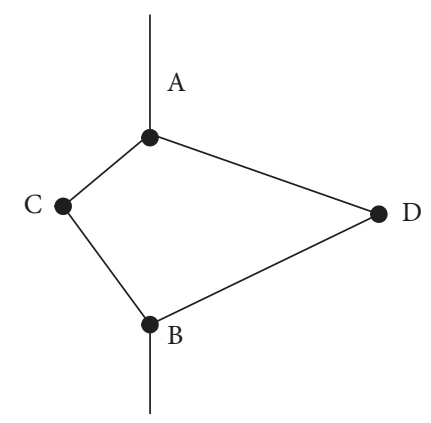

FIGURE 2: Intelligent water drops algorithm.

\section{Proposed Optimal Resource Allocation Scheme}

In this section, we proposed an improved algorithm to solve the optimal resource allocation formulated in (6).

3.1. Intelligent Water Drops Algorithm. Intelligent water drops (IWDs) algorithm is an intelligent algorithm introduced by Shah-Hosseini inspired by the flow of natural water drops, which construct a solution by cooperation with each other [18]. The IWDs are associated with two properties: the amount of soil in the path and the velocity of the IWDs. When the water drops pass through different paths, the change of the soil quantity on the different paths is different due to the difference of the path distance. When the subsequent water drops face different available paths, they are more likely to choose a path with less soil, in which the IWDs move faster. As shown in Figure 2, the initial amounts of soil at two paths are the same; when $t=0$, different intelligent water drops will select the paths $\mathrm{ACB}$ and $\mathrm{ADB}$ with the same probability. After the intelligent water drops pass these two paths, due to a shorter path of $\mathrm{ACB}$, the water drops selecting this path will run faster and carry a lot of soil, leading the soil on the path ACB to be less than that on ADB after the initial iteration. At $t=1$, the intelligent water drops will select a shorter path, ACB, with a greater probability. After repeated feedback at many times, the intelligent water drops will find the shortest path between A and B. The intelligent water drops algorithm draws on the feedback mechanism of changing soil quantity on the path and completes iterative search.

3.2. Improved Intelligent Water Drops Algorithm. The main drawback of the IWDs algorithm is the low speed at the early stage of training. Because the total amount of soil is the same on all paths, the intelligent water drops will randomly select a path, even if that path is not the optimal one; this will change the amount of soil on that path, resulting in the phenomenon of path dependence in the subsequent iteration process. Therefore, other water drops are inclined more to select that path and many invalid searching paths appear.

Genetic Algorithm (GA) [19] is an adaptive heuristic search algorithm based on the biological evolution process, reserving well-adapted individuals in the process of crossover and mutation, and after several evolutions, the optimal solution of the objective function is obtained. It starts the iterative process in individual population, which makes it easy to achieve expansion and algorithm fusion. GA has the characteristics of implicit parallelism and strong global search ability [20], which can quickly seek the solution in search space without trapping into the local optimal solution. The local search occurs in GA when the value of gItermax is too small, and GA will stop iterative search without finding the optimal solution; then IWDs algorithm begins, thus affecting the searching efficiency. In practice, GA encounters premature convergence problems.

As mentioned previously, IWDs algorithm is prone to many ineffective searches in the early stage, and the local search ability of the GA is limited in later period. We proposed a novel algorithm to improve the IWDs algorithm with GA. That is, in the early stage of the process, the global search ability of the GA is applied to achieve the rapid optimal solution, which is used as the initial solution of the IWDs algorithm. Finally, the global optimal solution is obtained by the characteristics of fast convergence of IWDs algorithm.

The flowchart of improved IWDs algorithm is shown in Figure 3.

Two main problems in improved IWDs algorithm should be mentioned as follows.

(1) Value of Boundary Condition gIter $_{\max }$. In the calculation process, the convergence is different due to different scale of data. The boundary condition gIter $_{\max }$ of GA and IWDs algorithm should be determined by the population size. The local search occurs in GA when the value of gIter $_{\max }$ is too small, and GA will stop iterative search without finding the optimal solution; then the algorithm transfers to IWDs algorithm, thus affecting the searching efficiency. On the contrary, if the value of gIter $_{\text {max }}$ is too large, it leads to the slow convergence in GA due to redundant computing. Furthermore, the early maturing of GA causes the phenomenon of path dependence in IWDs algorithm.

According to the convergence analysis of GA based on the Markov chain model mentioned in [21], the value of $g$ Iter $_{\max }$ is expressed as

$$
g \text { Iter }_{\max }=\frac{N\left(f^{*}-f^{*}\left(X_{1}\right)\right)}{2 \sqrt{K} p_{c} p_{m} p_{s \min } f^{*}\left(X_{1}\right)},
$$

where $N$ and $K$ are the numbers of subcarriers and users, respectively. $p_{c}$ and $p_{m}$ are the probability of crossover and mutation, $p_{s \text { min }}$ is the minimum selection probability of the nonoptimal individual, $f^{*}\left(X_{1}\right)$ is the fitness value of the best individual in initial population, and $f^{*}$ is the best individual fitness value in current population.

(2) Intelligent Water Drops Soil Quantity Initialization. GA achieves an optimal solution and many relatively good solutions, which are received with different weights and are used for the soil initialization of intelligent water drops. That is,

$$
\operatorname{soil}(i, j)=\operatorname{Intsoil} *\left(1-L_{*}^{\alpha}-\sum_{\tau=1}^{3} L_{\tau}^{\beta_{\tau}}\right), \quad(i, j) \in L
$$




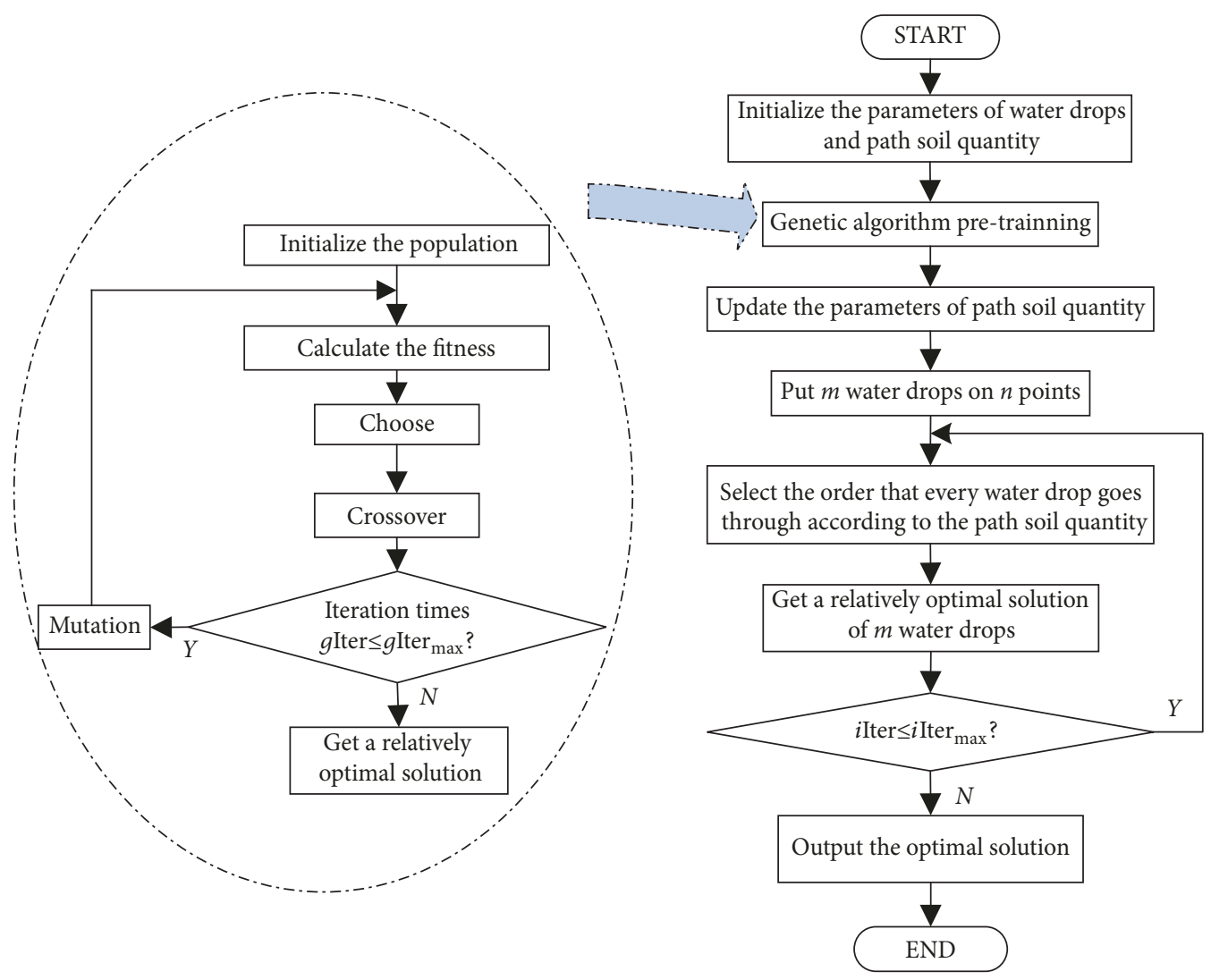

FIGURE 3: Flowchart of improved intelligent water drops algorithm.
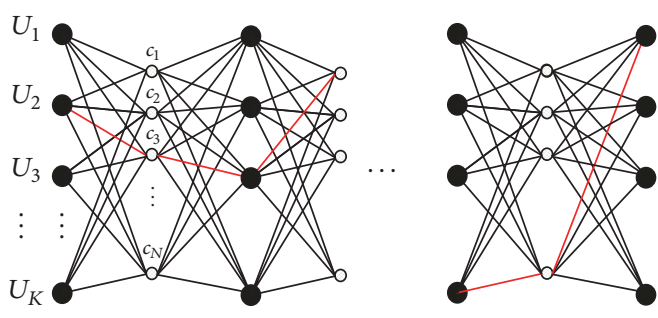

FIGURE 4: Two-level neural network model.

In particular, $L_{*}$ is the optimal solution generated by GA. $L_{\tau}(\tau=1,2,3)$ refers to the three optimal solutions generated by GA and sorted by the fitness value. $\alpha$ and $\beta_{\tau}$, respectively, refer to the weight of solution at the initialization.

\section{Optimal Resource Allocation Scheme}

4.1. Optimal Resource Allocation Model. The model in (6) is the combinatorial optimization problem, which should meet a series of continuous or discrete conditions to obtain the optimal resource allocation. So, we present two-level neural network model to solve the problems of resource allocation in cooperative communication system and then seek the optimal path with improved IWDs algorithm.

The two-level neural network model is shown in Figure 4. It is composed of two types of network nodes and the connection edges between adjacent nodes. The basic unit is rows. A row of $K$ master nodes represents the $K$ users and a row of $N$ secondary nodes represents the $N$ unallocated subcarriers in cooperative communication system. The user's primary nodes are a total of $K+1$ rows. A new row inserted between two adjacent rows represents secondary nodes of subcarriers. A path between two roles of primary nodes presents the two selected subcarriers, and then this path is weighted according to (6). From the first row to the $K+$ 1 column, a path passing $2 K$ nodes represents a possible allocation scheme. In this way, the optimal energy-efficient resource allocation in cooperative communication system transfers into the path planning in this two-level neural network. The red path in this figure represents a possible allocation scheme.

4.2. Optimal Resource Allocation Scheme. The optimal resource allocation flow of improved IWDs algorithm is described as follows.

Step 1. Initialize global static parameters; the amount of IWDs $N_{\text {IWD }}=K$.

Step 2. Pretrain Genetic Algorithm.

Step 2.1. Initialize the population and the static parameters.

Step 2.2. Calculate the population fitness according to (6).

Step 2.3. Operate the heredity and mutation on the population according to the boundary conditions of (8); operate the mutation and iteration until the end of loop. 
TABLE 1: Simulation parameters.

\begin{tabular}{lc}
\hline Parameters & Values \\
\hline Total system bandwidth $B$ & $15 \mathrm{MHz}$ \\
Number of subcarrier $N$ & 64 \\
Subcarrier mean signal to noise ratio & $38 \mathrm{~dB}$ \\
Path-loss factor & 3.5 \\
Maximum delay extension & $4 \mu \mathrm{m}$ \\
Maximum Doppler shift & $30 \mathrm{~Hz}$ \\
Channel status information update cycle & $0.5 \mathrm{~ms}$ \\
Maximum transmit power $P_{\max }$ & $30 \mathrm{dBm}$ \\
Circuit power $P_{c}$ & $27 \mathrm{dBm}$ \\
Transmitter power amplifier efficiency $1 / \xi$ & $38 \%$ \\
User minimum transfer rate $R_{\min }$ & $1.2 \mathrm{Mbps}$ \\
\hline
\end{tabular}

Step 3. Place $N_{\text {IWD }}$ water drops on primary nodes on the left as shown in Figure 4, and iterate each intelligent water drop according to Steps 4 and 5.

Step 4. Iterate intelligent water drops.

Step 4.1. Initialize the intelligent water drops' parameters and the soil quantity on the path according to (9).

Step 4.2. Empty the Tabu list, and list all subcarriers as assignable.

Step 4.3. With the Tabu list, calculate the probability of all selectable paths (subcarrier pair scheme), and select the most suitable subcarrier pair $(m, n)$ as the user's allocation scheme.

Step 4.4. Put the subcarrier $m$ in Tabu list 1 and subcarrier $n$ in Tabu list 2, which indicates that this subcarrier pair has been occupied by the system in two time slots.

Step 4.5. Update the amount of path soil and soil carried by the intelligent water drops.

Step 4.6. Intelligent water drops pass through the path to the next primary node; set the primary node as the initial position of the next path selection, repeat the steps from Step 4.3 to Step 4.5 until the water drops reach the model on the right side as shown in Figure 4, and this loop ends.

Step 5. At the end of the current iteration, calculate the optimal solution of all paths of water drops, and update the total amount of the path soil according to the optimal solution.

Step 6. Determine whether the number of iterations satisfies $i$ Iter $\leq i$ Iter $_{\max }$; if it does, then repeat Steps 4 and 5; otherwise, it goes to the end of program and optimal solution is shown.

\section{Numerical Analysis}

In this section, we evaluate the performance of the proposed optimal resource allocation scheme via simulation on MATLAB 2014b. The parameters are shown in Table 1.

5.1. Performance Analysis of Proposed Optimal Scheme. In Figure 5, if the number of users in system is 10 , the

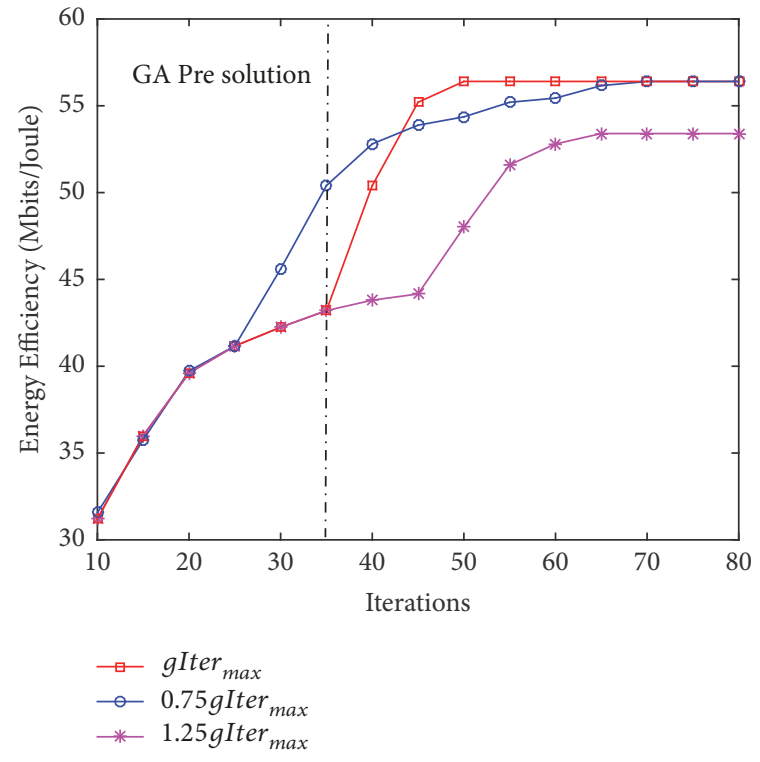

FIGURE 5: Energy efficiency comparison of different $g$ Iter $_{\max }$.

relationship between energy efficiency and $g$ Iter $_{\max }$ is depicted. The proposed algorithm is combined with GA and IWDs algorithm. That is, after pretraining by GA at early period of iterations, the performance is further improved by using IWDs at later period. It is obvious that when the value of boundary condition is $0.75 * \mathrm{gIter}_{\max }$, the premature convergence occurs. In this case, it is inefficient that IWDs are operated due to GA getting struck at local optimal solution. When the value of boundary condition is $1.25 *$ gIter $_{\text {max }}$, the performance declines, and the premature convergence of GA makes the phenomenon of path dependence in IWDs occur.

5.2. Performance Comparison of Variant Algorithms. Figure 6 illustrates the performance comparison of improved IWDs algorithm in terms of energy efficiency. Obviously, the energy efficiency obtained by employing the proposed algorithm is much higher than that of GA and Ant Colony Optimization (ACO) algorithm mentioned in [22]. That is, the better performance is achieved by using improved IWDs algorithm. 


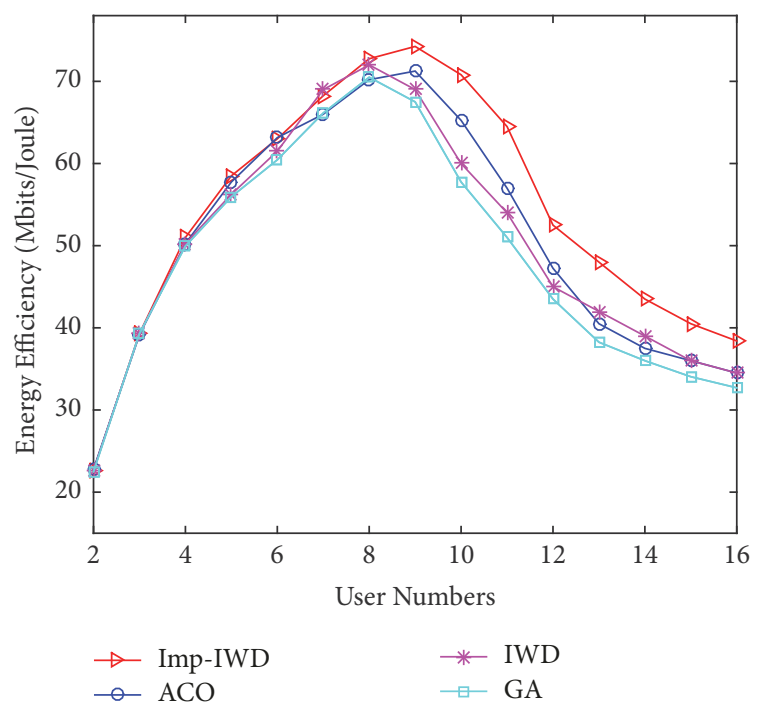

Figure 6: Energy efficiency comparison of different user numbers.

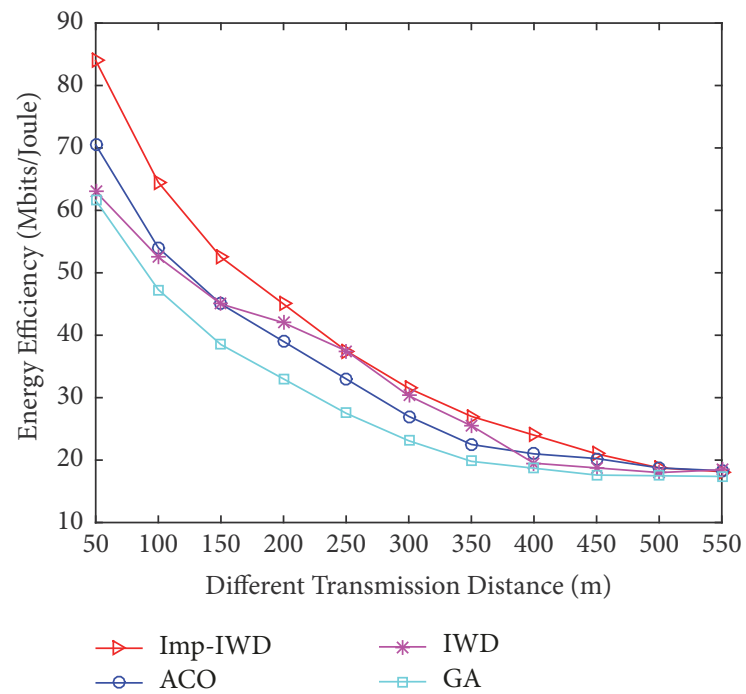

FIgURE 7: The system energy efficiency comparison of different transmission distance.

However, the algorithmic stability of IWDs is poor for its local optimum. Meanwhile, the performance increases at the beginning and then decreases, while the number of users increases. Due to limitation of system resources, when the number of users increases, it results in a lack of system resources and furthermore the performance degradation.

If the number of users is 10 , the radius is 600 meters. Figure 7 shows the relationship between energy efficiency and the distance between the users and BS, comparison of different resource allocation schemes. It is proven that the proposed improved IWDs algorithm obtains better performance than that of algorithms in [22]. At the same time, with the increasing of transmission distance, the energy efficiency is gradually reduced. When the transmission distance is far, the channel condition becomes more severe, so the

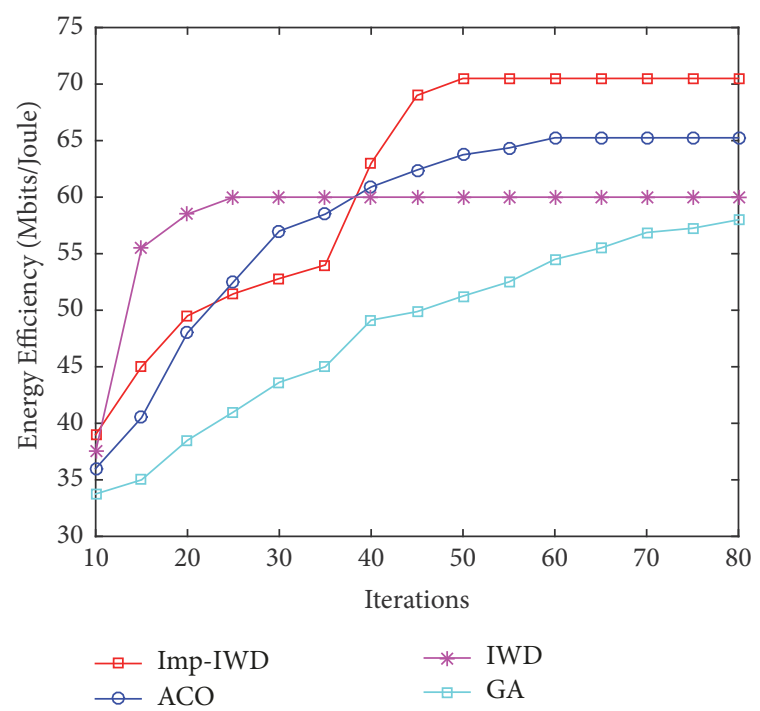

FIgURE 8: The convergence rate of different algorithms.

transmitting power increases to overcome the path loss, resulting in reduced system energy efficiency.

Figure 8 shows the convergence rate of different algorithms if the number of users is 10 . The improved IWDs algorithm has a faster convergence rate and obtains a better performance, while the original IWDs algorithm encounters premature convergence.

\section{Conclusion}

We have addressed the optimal resource allocation for uplink single-cell multiuser cooperative communication system. With the goal of optimizing energy efficiency, the improved IWDs algorithm has faster convergence rate, which achieves better resource allocation. The performance evaluation results demonstrate the effectiveness of the proposed solution.

It is important to notice that a subcarrier is assigned to a user only in this paper. In the practical communication scenario, users' data are abrupt and asymmetric. In the future, a real-time resource allocation strategy can be established to meet the demands of rapid development of mobile services.

\section{Data Availability}

The network parameter data used to support the findings of this study are included within the article.

\section{Conflicts of Interest}

The authors declare that there are no conflicts of interest regarding the publication of this paper.

\section{Acknowledgments}

This work was supported by the Natural Science Foundation of Guizhou Province (Grant no. [2016]1054), Joint Natural Science Foundation of Guizhou Province (Grant 
no. LH[2017]7226), Academic Talent Training and Innovation Exploration Project of Guizhou University (Grant no. [2017]5788), and Graduate Student Innovation Foundation of Guizhou University (Grant no. 2017015).

\section{References}

[1] G. Auer, V. Giannini, C. Desset, I. Godor, P. Skillermark, and M. Olsson, "How much energy is needed to run a wireless network," Wireless Communications, vol. 18, no. 5, pp. 40-49, 2011.

[2] Y. Zhao, Y. Z. Wang, and H. Y. Yang, "Introduction to green communication technologies," Information \& Communications, vol. 5, pp. 254-255, 2016.

[3] J. C. Xu, "Current Situation and Countermeasures of Energy Conservation and Emission Reduction in Telecommunication Industry in China," Modern Economic Information, vol. 7, p. 304, 2014.

[4] A. Sendonaris, E. Erkip, and B. Aazhang, "User cooperation diversity-part I: system description," IEEE Transactions on Communications, vol. 51, no. 11, pp. 1927-1938, 2003.

[5] J. N. Laneman, D. N. Tse, and G. Wornell, "Cooperative diversity in wireless networks: efficient protocols and outage behavior," IEEE Transactions on Information Theory, vol. 50, no. 12, pp. 3062-3080, 2004.

[6] J. C. Xiao, Development of Virtual Massive MIMO Channel Measurement System and Analysis of Large Scale Fading, Beijing Jiaotong University, 2016.

[7] L. Li and C. Chigan, "A Virtual MIMO based anti-jamming strategy for cognitive radio networks," in Proceedings of the IEEE International Conference on Communications, pp. 1-6, 2016.

[8] C. Y. Wong, R. S. Cheng, K. B. Letaief, and R. D. Murch, "Multiuser OFDM with adaptive subcarrier, bit, and power allocation," IEEE Journal on Selected Areas in Communications, vol. 17, no. 10, pp. 1747-1758, 1999.

[9] K. N. Pappi, P. D. Diamantoulakis, H. Otrok, and G. K. Karagiannidis, "Cloud compute-and-forward with relay cooperation," IEEE Transactions on Wireless Communications, vol. 14, no. 6, pp. 3415-3428, 2015.

[10] S. Yousaf, N. Javaid, U. Qasim, N. Alrajeh, Z. A. Khan, and M. Ahmed, "Towards reliable and energy-efficient incremental cooperative communication for wireless body area networks," Sensors, vol. 16, no. 3, 2016.

[11] H. Li, N. Jaggi, and B. Sikdar, "Relay scheduling for cooperative communications in sensor networks with energy harvesting," IEEE Transactions on Wireless Communications, vol. 10, no. 9, pp. 2918-2928, 2011.

[12] A. Doosti-Aref and A. Ebrahimzadeh, "Adaptive Relay Selection and Power Allocation for OFDM Cooperative Underwater Acoustic Systems," IEEE Transactions on Mobile Computing, vol. 17, no. 1, pp. 1-15, 2018.

[13] X. Yin Long, Research on Energy Efficiency Based Cooperative Communication System, 2014.

[14] V. Rodoplu and T. H. Meng, "Bits-per-joule capacity of energylimited wireless networks," IEEE Transactions on Wireless Communications, vol. 6, no. 3, pp. 857-864, 2007.

[15] W.-S. Lai, T.-H. Chang, and T.-S. Lee, "Distributed dynamic resource allocation for ofdma-based cognitive small cell networks using a regret-matching game approach," Game Theory Framework Applied to Wireless Communication Networks, 2016.

[16] Y. Xu, Z. Bai, B. Wang et al., "Energy-efficient power allocation scheme for multi-relay cooperative communications," in Proceedings of the International Conference on Advanced Communication Technology, pp. 260-264, IEEE, 2014.

[17] Z. Y. Liu, H. Tang, P. Mao, S. M. Liu, and L. Feng, "Relay selection in cooperative communication with bipartite graph," Application Research of Computers, vol. 4, 2018.

[18] H. Shah-Hosseini, "Problem solving by intelligent water drops," in Proceedings of the Evolutionary Computation, CEC , IEEE Congress, pp. 3226-3231, 2007.

[19] Y. J. Ma and W. X. Yun, "Research progress of genetic algorithm," Application Research of Computers, vol. 4, pp. 1201-1210, 2012.

[20] M. Pei, Ant Colony Optimization Algorithm in the Allocation of Cloud Computing Resources, Shandong Normal University, 2015.

[21] S. Q. Kuang, Parameter Adaptive Controlling and Convergence Theory for Genetic Algorithms, Central South University, 2009.

[22] A. Zainaldin, H. Halabian, and I. Lambadaris, "Joint Resource Allocation and Relay Selection in LTE-Advanced Network Using Hybrid Co-Operative Relaying and Network Coding," IEEE Transactions on Wireless Communications, vol. 15, no. 6 , pp. 4348-4361, 2016. 


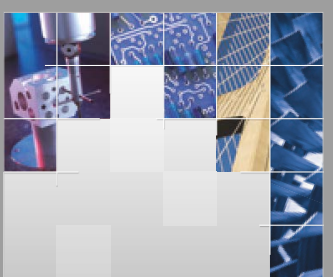

\section{Enfincering}
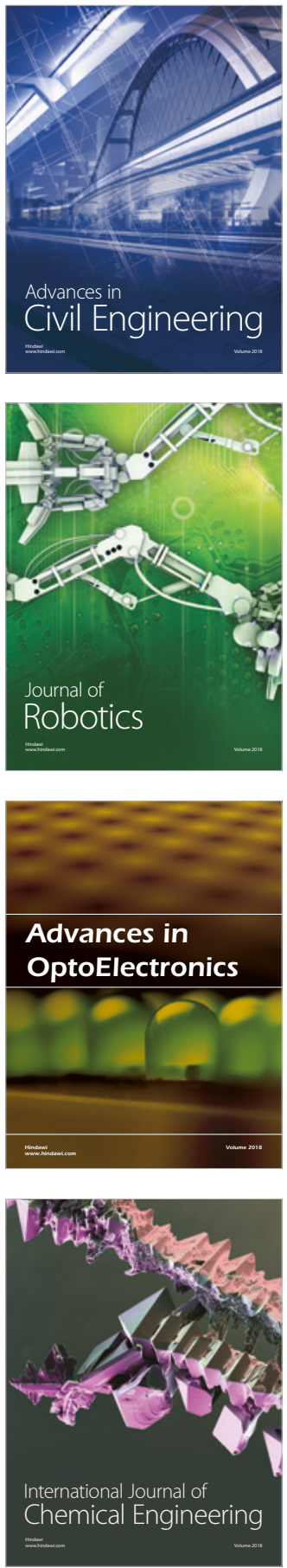

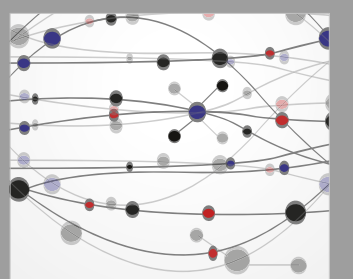

\section{Rotating \\ Machinery}

The Scientific World Journal

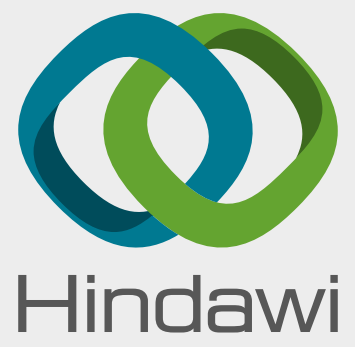

Submit your manuscripts at

www.hindawi.com
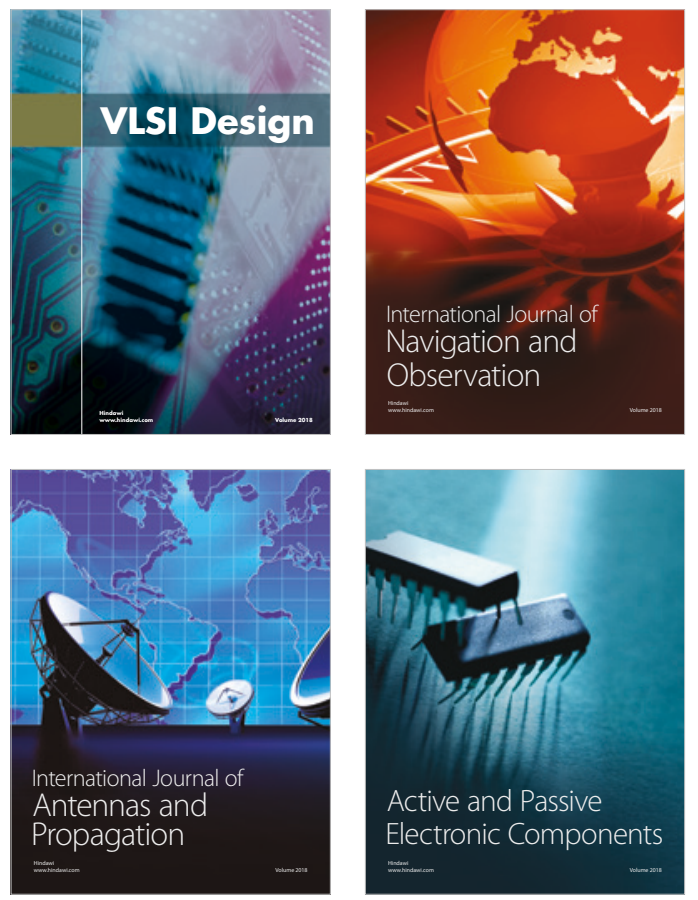
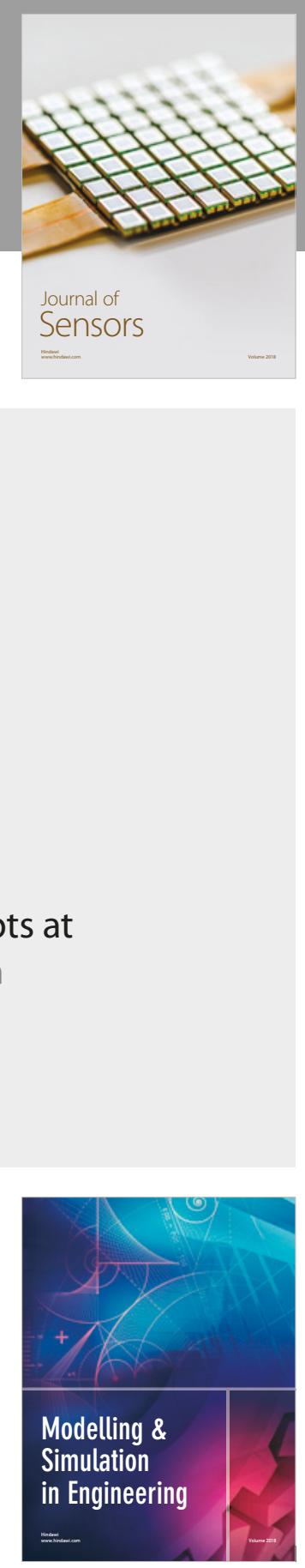

\section{Advances \\ Multimedia}
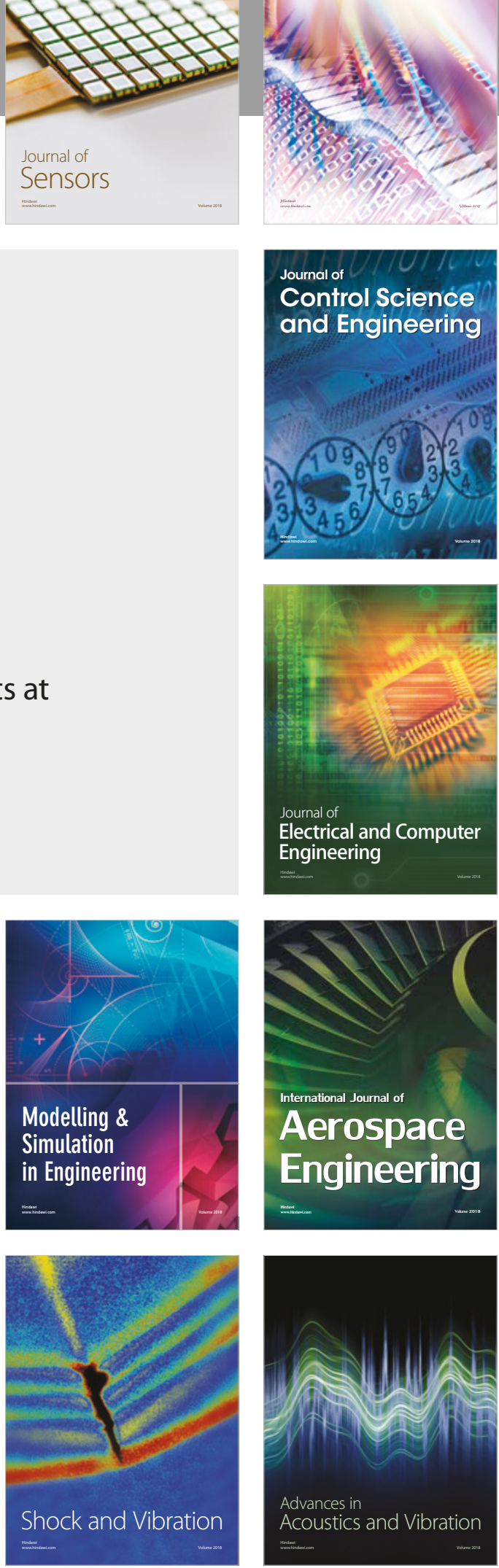